\title{
Video Surveillance System for Extra-high Voltage Power Transmission Lines with an Optimal Image Compression Method
}

\author{
Liyan Yuan ${ }^{1}$ and Huanbing $\mathrm{Gao}^{2}$ \\ ${ }^{1}$ Department of electronic engineering, Shandong College of Electric Technology, Jinan, China \\ ${ }^{2}$ School of information and electrical engineering, Shandong Jianzhu University, Jinan, China
}

\begin{abstract}
This paper presents a real-time online monitoring system scheme for power transmission line to achieve the objective of giving early warning to disaster and making decision for extra-high voltage power transmission line. Monitoring terminals with a S3C6410 ARM11 processor as core is equipped on the line towers to get the video data captured by the camera on board and other important information. An image optimal compression ratio is discussed based on JPEG2000 baseline compression in RGB space, and then a mathematical model for co-relationship of three color components in RGB is developed. The system has been installed on the power transmission line and works for a long time, and then it has indicated that the work efficiency of detecting the transmission line is raised.
\end{abstract}

Keywords-extra-high voltage power transmission line; image compression; ARM; $4 G$

\section{INTRODUCTION}

Over-head transmission lines is an important part of the power system, the failure of which would arouse serious negative effects, so there is very important significance in monitoring for the running state of transmission lines.

Due to the complex surrounding environment and the longdistance line of extra-high voltage power transmission lines, it will be the most beneficial for the development of power system automation transmitting information of transmission lines by wireless technology, especially the mobile communication technology. Communication networks of $4 \mathrm{G}$ are becoming increasingly mature nowadays, and the highspeed data transmission speed of $4 \mathrm{G}$ can satisfy the requirement of large amounts of information data transmission of power system[1]. There are many results in monitoring for transmission lines, but most of their communications are by GSM, GPRS, or $3 \mathrm{G}$ rather than $4 \mathrm{G}[2]$. Therefore, developing a video surveillance and state collection system for extra-high voltage power transmission lines based on $4 \mathrm{G}$ communication technology is essential and advanced in technology.

\section{EASE OF USE}

\section{A. Overall of the System}

The main constituent part of the system is video surveillance for transmission lines, which can record the manmade destroying, foreign objects attaching to the lines, swing of the lines, conductor icing, etc.. The video data captured by the camera are transmitted to monitoring host, and coded and integrated with grounding resistance of the towers, the swing information of the lines and the micro-meteorology received by Zigbee. The integrated data are sent to transmission lines monitoring center through $4 \mathrm{G}$ networks, or through GPRS if the signal of $4 \mathrm{G}$ is too weak. Therefore, the staff can obtain the scene and conditions of the transmission lines field so that they can make accurate estimates and exact judgments. The architecture diagram of the whole system is shown as Fig.1.

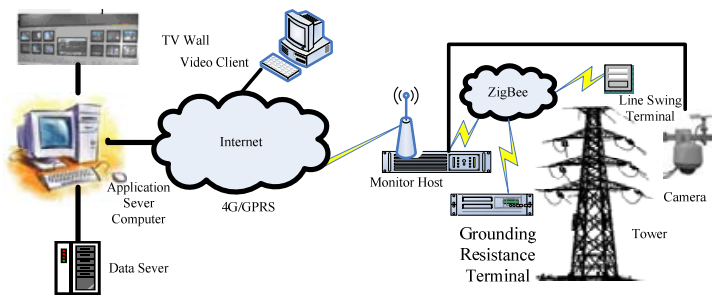

FIGURE I. STRUCTURE OF THE SYSTEM

\section{Design of Surveillance Terminal Host}

\section{A. Framework for the Surveillance Terminal Host}

A kind of high-performance embedded microprocessor and embedded real-time operating system are necessary to meet the requirements of capturing and compressing the video data, and communications by 4G and Zigbee. The Samsung S3C6410 is one of the highest performance mobile processors available today. This 32-bit ARM11 RISC microprocessor with AXI 64bit bus delivers up to $667 \mathrm{MHz}$ of processing performance. This sophisticated processor enables the integration of various functions - wireless communication, navigation, camera, gaming, music/video playing, mobile TV and PDA applications - into one device. An integrated Multi-Format Codec allows video capture and playback at as well as real-time video conferencing, with support of 5.1-channel Dolby sound. However, the S3C6410 also extends battery life because it was built using Samsung's $65 \mathrm{~nm}$ low-power process so the surveillance terminal host equipped on transmission line tower can be powered by solar energy or induction. The hardware architecture diagram is shown in Fig.2. 


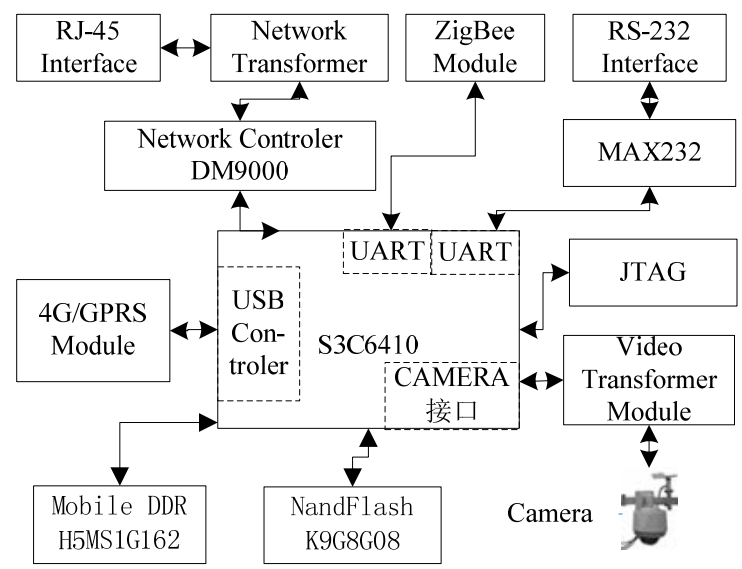

FIGURE II. HARDWARE ARCHITECTURE DIAGRAM

\section{B. Hardware of the Surveillance Terminal Host}

The clearness of the image, the transmission speed and the data traffic should be considered when designing the video capturing module. $4 \mathrm{G}$ is the fourth generation of mobile telecommunications technology. ITU-R specified a set of requirements for $4 \mathrm{G}$ standards, setting peak speed requirements for $4 \mathrm{G}$ service at $100 \mathrm{Mbit} / \mathrm{s}$ for high mobility communication and $1 \mathrm{Gbit} / \mathrm{s}$ for low mobility communication, which is enough for requirement of the clearness of the image and the transmission speed. But the data traffic would be lager if the resolution of image is higher.

After comparing the results of lot of experiments, the DELV-XCAM-ED5 camera is selected, which uses a $1 / 3$ SONY HQ1 CCD imaging element to provide a $512 \mathrm{H}$ x $492 \mathrm{~V}$ effective pixel image. The camera can ensure the resolution of the captured image and online traffic saving can made by setting from the condensing program running is the terminal host.

The TVP5147M1 device is a high-quality, single-chip digital video decoder that digitizes and decodes all popular baseband analog video formats into digital component video. The TVP5147M1 decoder supports the analog-to-digital (A/D) conversion of component $\mathrm{YPbPr}$ signals, as well as the $\mathrm{A} / \mathrm{D}$ conversion and decoding of NTSC, PAL, and SECAM composite and S-video into component $\mathrm{YCbCr}$. The TVP5147M1 decoder includes methods for advanced vertical blanking interval (VBI) data retrieval. A built-in FIFO stores up to 11 lines of teletext data, and with proper host port synchronization, full-screen teletext retrieval is possible. The TVP5147M1 decoder can pass through the output formatter $2 \times$ sampled raw luma data for host-based VBI processing.

\section{Communication Module of $4 G$}

ME3760 Module is a market proven $4 \mathrm{G}$ product offering LTE CAT3, designed for M2M applications with the demand for reliability and highest data speed. It supports TD-LTE, LTE FDD, TD-SCDMA. Video data are packed as RTP data, and then packed into UDP data packet to communication in the IP networks. RTP is a kind of real-time communication protocol above TCP AND UDP providing the service of point to point, suit for the real-time data transmitted by IP multicast and demand such as video data and audio data. There are some standards for time stamp, serial number, data type, and source identification in RTP protocol. In this system, the compressed video data of power tower field is packet into RTP data package and it is set as a regular event to sending the packet data. The other basic information of the transmission lines, grounding resistance of the towers, the swing information of the lines and the micro-meteorology, are transmitted by TCP protocol, with the terminal host as a TCP server. The monitor center can make a TCP client to connect every terminal host. And all the information are encrypted to ensure the safety.

\section{Method of IMAGE COMPRESSION}

\section{Method for moving image compression}

Lossless compression is adequate when low compression ratios are acceptable. However, substantial compression ratios can only be achieved with lossy compression schemes. The aim of image compression is to encode images or image sequences into as few bits as possible with a decoding mechanism that reconstructs the original image with an acceptable visual and/or informational quality. Another issue in image compression and decompression is its speed, especially in the monitor system for power transmission lines, which is an application requiring low cost traffic and real-time conveying the image without additional delay.

A simplified schematic representation of a method for moving image compression is shown in Fig. 3.

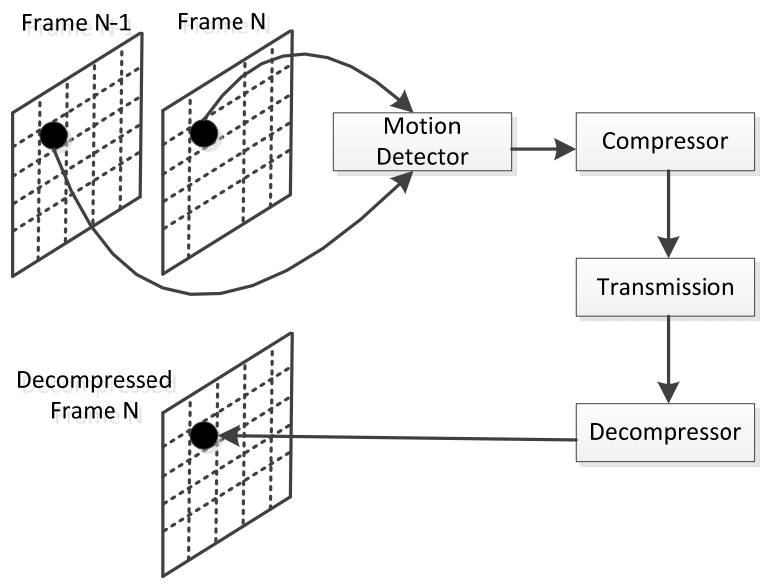

FIGURE III. DIAGRAM OF A VIDEO COMPRESSION SCHEME

A digital image $\mathrm{I}$ is described by a function

$\mathrm{f}: \mathrm{Z} \times \mathrm{Z} \rightarrow\{0,1, \ldots, 2 \mathrm{k}-1\}$,

where $\mathrm{Z}$ is the set of natural numbers and $\mathrm{k}$ is the maximum number of bits to be used to represent the gray level of each pixel.

In other words, $f$ is a mapping from discrete spatial coordinates $(\mathrm{x}, \mathrm{y})$ to gray-level values. Thus, $\mathrm{M} \times \mathrm{N} \times \mathrm{k}$ bits are required to store an $\mathrm{M} \times \mathrm{N}$ digital image. The aim of digital image compression is to develop a scheme to encode the original image I into the fewest number of bits so that the image I' reconstructed by decoding from this reduced 
representation is as similar to the original image as possible[3]. The problem is to design a compress and a decompress block so that $|\mathrm{Ic}|<<|\mathrm{I}|$ where $|$.$| denotes the size in bits.$

In lossy compression, the peak signal-to-noise ratio (PSNR) is often used as the measure of similarity or of dissimilarity, although it does not always reflect perceived visual quality as well as one would like. For moving images, the compression ratio may vary dynamically with the specific image or image portion being transmitted, since advantage is taken of the existence or nonexistence of significant motion in successive image frames. However, the PSNR metric can still be used to compare corresponding frames in the original and decompressed image sequences[4].

Let the original and reconstructed images be denoted by functions $\mathrm{f}(\mathrm{x}, \mathrm{y})$ and $\mathrm{g}(\mathrm{x}, \mathrm{y})$ of the pixel plane position $(\mathrm{x}, \mathrm{y})$, respectively. The PSNR for the reconstructed image $g(x, y)$ is defined by:

$$
P S N R=10 \lg _{10} \frac{\left(2^{K}-1\right)^{2}}{e_{r m s}^{2}},
$$

where

$$
e_{r m s}^{2}=\overline{e^{2}}=\frac{1}{M N} \sum_{x=0}^{M-1} \sum_{y=0}^{N-1}[g(x, y)-f(x, y)]^{2}
$$

\section{A. JPEG2000 Baseline Compression}

To get a high transmitting speed, the image captured by the camera should be compressed. Many standard generic compression techniques have effectively been employed for image compression. A linear transform is used to obtain a representation of the input image in the domain. A quantization is carried out on the transform coefficients in order to smooth out high frequencies. As with most of image compression algorithms, JPEG2000 is composed of a wavelet transform stage followed by a quantization stage and an entropy coding stage. The first step of the JPEG2000 encoder is the color transform to RGB space, which can also transform to other color spaces[5,6].

Consider a $\mathrm{M} \times \mathrm{N}$ image $X(X \in\{R, G, B\})$ in RGB space and let $x(i, j) \in\{r(i, j), g(i, j), b(i, j)\}$ denote the $\mathrm{R}, \mathrm{G}, \mathrm{B}$ values of pixel at $(i, j)$ of the original image, then the image $\mathrm{X}$ with wavelet coefficient can be written as:

$$
X=\{x(i, j) \mid i=0,1, \cdots, M-1 ; j=0,1, \cdots, N-1\}
$$

After n-level wavelet decomposition by $(9,7)$ wavelet filter [2], a new wavelet coefficient of image $\mathrm{X}$ can be obtained:

$$
\tilde{X}=\{\tilde{x}(i, j) \mid i=0,1, \cdots, M-1 ; j=0,1, \cdots, N-1\}
$$

The final distortion value $\mathrm{D}$ is the statistical average of

$$
D=E[d(\tilde{X}, \hat{X})]=E(\tilde{x}(k, l), \hat{x}(k, l))=T^{2} / 2
$$

As the high frequency sub-band is a non-memory Gaussian source [3] and the relationship between its Distortion D, then the Bit rate $\mathrm{R}$ is:

$$
R(D)=H(X)-\frac{1}{2} \log _{2}^{2 \pi e D}
$$

where e is the Euler's number,

$H(x)=\int_{-\infty}^{+\infty} p(x) \log _{2} p(x)$ is the entropy of Gaussian source and its value is related to the image. However, the result is irrelevant to the selection of quantization step T. After wavelet decomposition and EBCOT (Embedded block Coding with Optimized Truncation), the whole code (bitstream) length can be obtained:

$$
L=L_{L L n}+\sum_{i=1}^{n}\left(L_{L H i}+L_{H L i}+L_{H H i}\right)
$$

where LHi, HLi, HHi and LLn are sub-bands, and $L_{L H i}$, $L_{H L i}, L_{H H i}$ and $L_{L L n}$ are their code length, respectively. Note that $L_{L L n}$ takes a small proportion in the total code length $L$ and its variation has little effect on $\mathrm{L}$. Therefore, the value of $L_{L L n}$ is treated as a fixed value for the sake of convenience.

Substitute Eq. (6) and (7) to (8), the relationship between $L_{L H i}$ and distortion $\mathrm{D}$ can be described as:

$$
L_{L H i}=\frac{M \times N}{4^{i}} \times R_{L H i}=\frac{M \times N}{4^{i}}\left(H_{L H i}-\frac{1}{2} \log _{2}^{\pi e T^{2}}\right)
$$

where $R_{L H i}$ is the bit rate of the sub-band LHi, $H_{L H i}$ is the entropy of the sub-band LHi . Similarly, the relationship between $L_{H L i}$ and $L_{H H i}$ and Distortion D can also be acquired as:

$$
\begin{aligned}
L_{H L i} & =\frac{M \times N}{4^{i}}\left(H_{H L i}-\frac{1}{2} \log _{2}^{\pi e T^{2}}\right) \\
L_{H H i} & =\frac{M \times N}{4^{i}}\left(H_{H H i}-\frac{1}{2} \log _{2}^{\pi e T^{2}}\right)
\end{aligned}
$$

Eq.(7) can be further deducted as:

$$
\begin{aligned}
& L=L_{L L n}+\sum_{i=1}^{n} \frac{M \times N}{4^{i}}\left(H_{L H i}+H_{H L i}+H_{H H i}\right) \\
& +M \times N \times\left(1-\left(\frac{1}{4}\right)^{n}\right) \times\left(-\frac{1}{2} \log _{2}^{e \pi T^{2}}\right)
\end{aligned}
$$


Then the calculation method of the code length of these three components of a color image can be obtained. The code length $L^{r}$ of red component is:

$$
\begin{aligned}
& L^{r}=L_{L L n}^{r}+\sum_{i=1}^{n} \frac{M \times N}{4^{i}}\left(H_{L H i}^{r}+H_{H L i}^{r}+H_{H H i}^{r}\right) \\
& +M \times N \times\left(1-(1 / 4)^{n}\right) \times\left(-\frac{1}{2} \log _{2}^{\pi e T_{r}^{2}}\right)
\end{aligned}
$$

The code length $L^{g}$ of green component is:

$$
\begin{aligned}
& L^{g}=L_{L L n}^{g}+\sum_{i=1}^{n} \frac{M \times N}{4^{i}}\left(H_{L H i}^{g}+H_{H L i}^{g}+H_{H H i}^{g}\right) \\
& +M \times N \times\left(1-(1 / 4)^{n}\right) \times\left(-\frac{1}{2} \log _{2}^{\pi e T_{g}^{2}}\right)
\end{aligned}
$$

The code length $L^{b}$ of blue component is:

$$
\begin{aligned}
& L^{b}=L_{L L n}^{b}+\sum_{i=1}^{n} \frac{M \times N}{4^{i}}\left(H_{L H i}^{b}+H_{H L i}^{b}+H_{H H i}^{b}\right) \\
& +M \times N \times\left(1-(1 / 4)^{n}\right) \times\left(-\frac{1}{2} \log _{2}^{\pi e T_{b}^{2}}\right)
\end{aligned}
$$

where $T_{r}, T_{g}$ and $T_{b}$ are the quantization steps of red, green and blue components, respectively. $L_{L L n}^{k}, H_{L H i}^{k}, H_{H L i}^{k}$ and $H_{H L i}^{k}$ are the code stream length of sub-band LLn, entropy of the sub-bands LHi, HLi, HHi of each three component respectively, where $k \in\{r, g, b\}$ delegates red, green and blue colors[7,8].

Based on the calculation method of color image quality, the color error $C_{\varepsilon i}$ of each pair pixel between input and output can be calculated as:

$$
C_{\varepsilon i}=\left[\begin{array}{l}
u \times\left(r_{(\text {in }) i}-r_{(\text {out }) i}\right)^{2}+v \times\left(g_{(\text {in }) i}-g_{(\text {out }) i}\right)^{2} \\
+w \times\left(b_{(\text {in }) i}-b_{(\text {out }) i}\right)^{2}
\end{array}\right]^{1 / 2}
$$

where $u=0.089401, v=0.344569$ and $w=0.012996$ Furthermore, the color error of each rectangular block is:

$$
\begin{aligned}
T M_{C} & =\alpha^{-} \sum_{i=1}^{\alpha} C_{\varepsilon i}=\alpha^{-} \sum_{i=1}^{\alpha} E\left[C_{\varepsilon i}\right] \\
& =\left(\frac{u}{2} \times T_{r}^{2}+\frac{v}{2} \times T_{g}^{2}+\frac{w}{2} \times T_{b}^{2}\right)^{1 / 2}
\end{aligned}
$$

where $\alpha$ is the number of the data in each rectangular block. If given the total code length of the color image $\mathrm{L}$, the $T M_{C}$ has to be minimized. Lagrange multiplier method is here used to seek the optimal solution.

The color error is reduced and the compressive quality is enhanced by using this method for compression.

\section{MOnitoring Center Software}

The online monitoring center software is developed with C\# language and ADO.NET database access technology and designed as $\mathrm{C} / \mathrm{S}$ architecture. The software is running on Windows Server 2003, and it is including 4 functional module: system management, site management, data analysis, video monitoring. The main GUI of the software is shown in Fig.4.

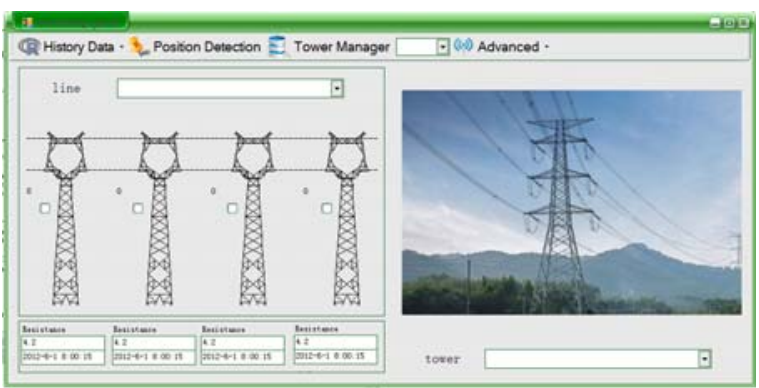

FIGURE IV. MAIN GUI OF THE SOFTWARE

\section{CONCLUSIONS}

In this paper, a video surveillance and state collection system for extra-high voltage power transmission lines is presented, the data of which is transmitted by $4 \mathrm{G}$ communication technology. The surveillance terminal host is designed with an ARM chip as its processor. To obtain higher compression ratios for saving $4 \mathrm{G}$ traffic and improving the communication speed, a method among three color components in RGB space is proposed. VDR test methods combined with entropy are used to evaluate the optimal compression ratio. This system is equipped on a part of extrahigh voltage power transmission lines in Shandong Province, China, and the early warning system for transmission networks has been initially formed.

\section{ACKNOWLEDGMENT}

This work is partially supported by the College Head Teachers Domestic Visiting Scholar Project of Shandong.

\section{REFERENCES}

[1] Li Yongliang, Zhang Tao, Luo Hong, et.al. Application of Wireless Private Network in Ningxia $330 \mathrm{kV}$ Trans mission Line Monitoring. Electrical Automation. 2014, Vol.36, No.2, pp.39-42.

[2] Zhao Jian-qing, Yao Yao, Qiu Wanhui, et.al. Study on intelligent early warning system based on transmission line on-line inspection system. Power System Protection and Control. 2013, Vol.41, No.23:49-54.

[3] LI Gang, QIU Shang-bin, LIN Ling, et al. New moving target detection method based on background differ-encing and coterminous frames differencing. Chinese Journal of Scientific Instrument, 2006, Vol.27, No.8, pp.961-964. 
[4] Farzaneh M, Savadjiev K. Statistical analysis of field data for precipitation icing accretion on overhead power lines. IEEE Trans on Power Delivery, 2005, Vol.20, No.2, pp.1080-1087.

[5] HUANG Xinbo. Transmission lines on linemonitoring and fault diagnosis. Beijing, China:China Elecmc Power Press, 2008.

[6] JIN Lijun, HU Juan, YAN Shujia. Method of spacer fault diagnose on transmission line based on image processing. High Voltage Engineering, 2013, Vol.39, No.5, pp.1040-1045.

[7] Raguram. R, Marcellin. M. W and Bilgin. A, mproved Resolution Scalability for Bilevel Image Data in JPEG2000, IEEE Transactions on Image Processing, 2009, Vol. 18, No. 4, pp. 774-782.

[8] Raguram. R, Marcellin. M. W and Bilgin. A, Improved Resolution Scalability for Bilevel Image Data in JPEG2000, IEEE Transactions on Image Processing, Vol. 18, No. 4, pp. 774-782, 2009. 